\title{
Folate Receptor $\beta$-Targeted PET Imaging of Macrophages in Autoimmune Myocarditis
}

\author{
Arghavan Jahandideh ${ }^{1,2}$, Sauli Uotila ${ }^{1}$, Mia Ståhle ${ }^{1}$, Jenni Virta ${ }^{1}$, Xiang-Guo Li ${ }^{1,3}$, Ville Kytö ${ }^{4}$, Päivi Marjamäki ${ }^{1}$, \\ Heidi Liljenbäck ${ }^{1,5}$, Pekka Taimen ${ }^{6}$, Vesa Oikonen ${ }^{1}$, Jukka Lehtonen ${ }^{7}$, Mikko I. Mäyränpää8, Qingshou Chen ${ }^{9}$, \\ Philip S. Low ${ }^{9}$, Juhani Knuuti ${ }^{1,2}$, Anne Roivainen ${ }^{1,2,5}$, and Antti Saraste ${ }^{1,2,4}$ \\ ${ }^{I}$ Turku PET Centre, University of Turku, Turku, Finland; ${ }^{2}$ Turku PET Centre, Turku University Hospital, Turku, Finland; ${ }^{3}$ Turku PET \\ Centre, Åbo Akademi University, Turku, Finland; ${ }^{4}$ Heart Center, Turku University Hospital and University of Turku, Turku, Finland; \\ ${ }^{5}$ Turku Center for Disease Modeling, University of Turku, Turku, Finland; ${ }^{6}$ Institute of Biomedicine, University of Turku and \\ Department of Pathology, Turku University Hospital, Turku, Finland; ${ }^{7}$ Heart and Lung Center, Helsinki University and Helsinki \\ University Hospital, Helsinki, Finland; ${ }^{8}$ Pathology, Helsinki University and Helsinki University Hospital, Helsinki, Finland; and \\ ${ }^{9}$ Department of Chemistry, Purdue University, West Lafayette, Indiana
}

Currently available imaging techniques have limited specificity for the detection of active myocardial inflammation. Aluminum ${ }^{18} \mathrm{~F}$-labeled 1,4,7-triazacyclononane- $N, N^{\prime}, N^{\prime \prime}$-triacetic acid conjugated folate $\left({ }^{18} \mathrm{~F}\right.$ FOL) is a PET tracer targeting folate receptor $\beta$ (FR- $\beta$ ), which is expressed on activated macrophages at sites of inflammation. We evaluated ${ }^{18} \mathrm{~F}-\mathrm{FOL} \mathrm{PET}$ for the detection of myocardial inflammation in rats with autoimmune myocarditis and studied the expression of FR- $\beta$ in human cardiac sarcoidosis specimens. Methods: Myocarditis was induced by immunizing rats $(n=18)$ with porcine cardiac myosin in complete Freund adjuvant. Control rats $(n=6)$ were injected with Freund adjuvant alone. ${ }^{18} \mathrm{~F}-\mathrm{FOL}$ was intravenously injected, followed by imaging with a small-animal PET/CT scanner and autoradiography. Contrastenhanced high-resolution CT or ${ }^{18} \mathrm{~F}-\mathrm{FDG}$ PET images were used for coregistration. Rat tissue sections and myocardial autopsy samples from 6 patients with cardiac sarcoidosis were studied for macrophages and FR$\beta$. Results: The myocardium of 10 of 18 immunized rats showed focal macrophage-rich inflammatory lesions, with FR- $\beta$ expression occurring mainly in M1-polarized macrophages. PET images showed focal myocardial ${ }^{18} \mathrm{~F}-\mathrm{FOL}$ uptake colocalizing with inflammatory lesions $\left(\mathrm{SUV}_{\text {mean }}\right.$, $2.1 \pm 1.1$ ), whereas uptake in the remote myocardium of immunized rats and controls was low (SUV mean, $0.4 \pm 0.2$ and $0.4 \pm 0.1$, respectively; $P<$ $0.01)$. Ex vivo autoradiography of tissue sections confirmed uptake of ${ }^{18} \mathrm{~F}-\mathrm{FOL}$ in myocardial inflammatory lesions. Uptake of ${ }^{18} \mathrm{~F}-\mathrm{FOL}$ in inflamed myocardium was efficiently blocked by a nonlabeled FR- $\beta$ ligand folate glucosamine in vivo. The myocardium of patients with cardiac sarcoidosis showed many FR- $\beta$-positive macrophages in inflammatory lesions. Conclusion: In a rat model of autoimmune myocarditis, ${ }^{18} \mathrm{~F}-\mathrm{FOL}$ shows specific uptake in inflamed myocardium containing macrophages expressing FR- $\beta$, which were also present in human cardiac sarcoid lesions. Imaging of FR- $\beta$ expression is a potential approach for the detection of active myocardial inflammation.

Key Words: folate receptor; PET; myocarditis; experimental autoimmune myocarditis

J Nucl Med 2020; 61:1643-1649

DOI: 10.2967/jnumed.119.241356

Received Dec. 23, 2019; revision accepted Feb. 26, 2020.

For correspondence or reprints contact: Antti Saraste, Heart Center, Turku University Hospital, Hämeentie 11, Fl-20520 Turku, Finland.

E-mail: antti.saraste@utu.fi

Published online Apr. 13, 2020.

COPYRIGHT (C 2020 by the Society of Nuclear Medicine and Molecular Imaging.
$\mathbf{M}$ terized by myocardial inflammatory lesions and myocyte necrosis resulting from infection, cardiotoxic agents, or autoimmune diseases such as sarcoidosis. The clinical sequelae of myocarditis include conduction disturbances, ventricular arrhythmias, and inflammatory cardiomyopathy, defined as myocarditis associated with cardiac dysfunction (1).

Diagnosis of myocarditis is challenging because of its wide clinical spectrum and nonspecific presentation. Endomyocardial biopsy is the gold standard for diagnosing myocarditis, but it has low sensitivity because of the focal nature of the disease (2). Thus, advanced noninvasive cardiac imaging, including cardiac MR and PET/CT with ${ }^{18}$ F-FDG, plays an important role in defining myocardial abnormalities $(2,3)$.

Although ${ }^{18} \mathrm{~F}-\mathrm{FDG} \mathrm{PET} / \mathrm{CT}$ has shown high accuracy for the detection of cardiac inflammation and provides prognostic information $(4,5)$, incomplete suppression of physiologic myocardial ${ }^{18}$ F-FDG uptake may impair its diagnostic accuracy (6). Therefore, more specific tracers for the detection of myocarditis on PET have recently been investigated (7-12).

Folate receptor $\beta$ (FR- $\beta$ ) is a glycosylphosphatidylinositolanchored membrane protein expressed on activated macrophages (13). Expression of FR- $\beta$ has been found in many inflammatory diseases, including rheumatoid arthritis, Crohn disease, ulcerative colitis, pulmonary disease, interstitial pneumonia, systemic lupus erythematosus, psoriasis, scleroderma, sarcoidosis, atherosclerosis, and myocardial infarction (13-16). Radiolabeled folate derivatives have been used to assess FR- $\beta$-positive activated macrophages at the site of inflammation, thereby suggesting FR- $\beta$ as a potential target for diagnosis of inflammatory diseases $(13,15)$. However, the feasibility of FR- $\beta$-targeted imaging has not been studied in myocarditis.

We evaluated the feasibility of the recently developed FR$\beta$-targeting radiotracer aluminum ${ }^{18} \mathrm{~F}$-labeled 1,4,7-triazacyclononane$N, N^{\prime}, N^{\prime \prime}$-triacetic acid conjugated folate $\left({ }^{18} \mathrm{~F}-\mathrm{FOL}\right)(15)$ for PET imaging of myocardial inflammation in a rat model of autoimmune myocarditis. Myocarditis was induced by immunization of rats with cardiac myosin in complete Freund adjuvant. Myocardial uptake of ${ }^{18}$ F-FOL was evaluated using small-animal PET/CT imaging and ex vivo autoradiography. Expression of FR- $\beta$ in 


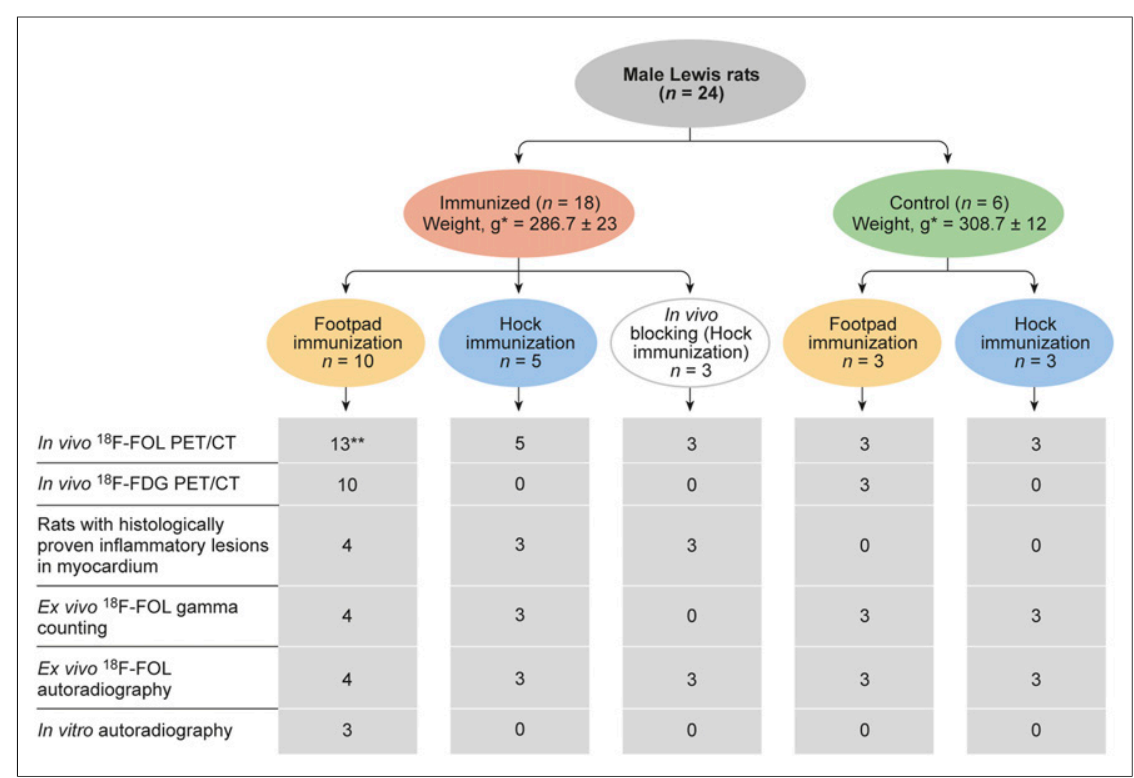

FIGURE 1. Flow diagram of study design and numbers of animals used. Blocking study $=$ in vivo competition assay with 100-fold molar excess of folate glucosamine injected 10 min before ${ }^{18} \mathrm{~F}-\mathrm{FOL} .{ }^{\star}$ Values are mean $\pm \mathrm{SD}$. ${ }^{\star \star} T e n$ had static scan, and all others had dynamic scan.

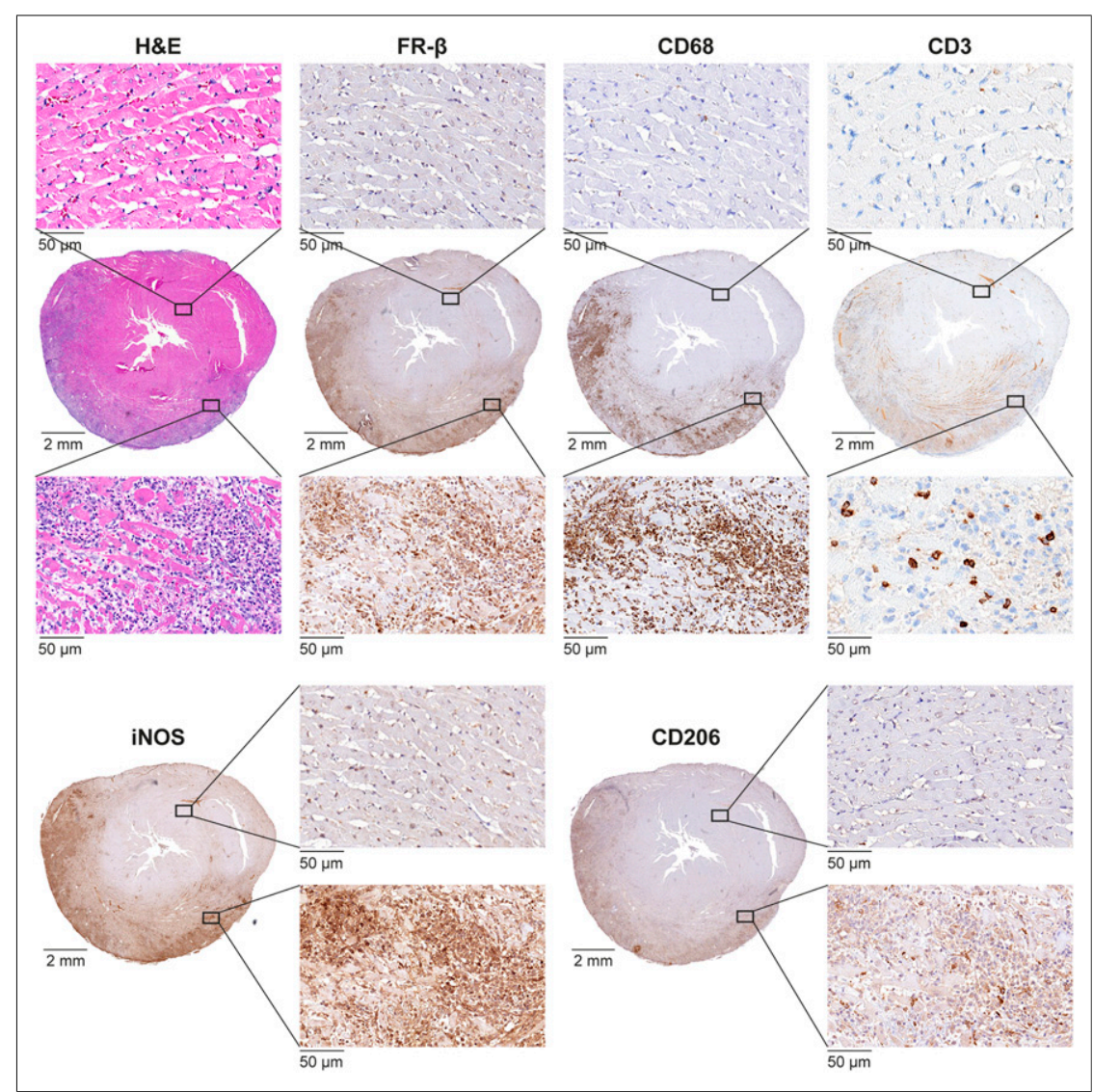

FIGURE 2. Inflammatory myocardial lesion in rat with autoimmune myocarditis identified by hematoxylin and eosin (H\&E) staining and stained with antibody against FR- $\beta$, which is colocalized with CD68-positive macrophages stained with antibody against CD68. Scattered CD3-positive lymphocytes are also present. Staining with antibodies against inducible nitric oxide synthase (iNOS) indicates presence of M1-polarized macrophages and, to a lesser extent, presence of M2-polarized macrophages stained with antibodies against CD206. myocardial inflammatory lesions was studied in rat hearts and in human cardiac sarcoidosis specimens.

\section{MATERIALS AND METHODS}

\section{Animal Model and Study Protocol}

Autoimmune myocarditis was induced as previously described $(17,18)$. Briefly, male Lewis rats received subcutaneous $5 \mathrm{mg} / \mathrm{mL}$ injections of pig cardiac myosin (M0531; Sigma Aldrich) in an equal volume of complete Freund adjuvant supplemented with Mycobacterium tuberculosis (1 mg/mL, F5881; Sigma Aldrich), with 2 injections being given $7 \mathrm{~d}$ apart, into either the footpad or the hock of the left foot. To enhance immunization after the hock injection, the rats also received an intraperitoneal 250 $\mathrm{ng} / \mathrm{mL}$ injection of pertussis toxin (P2980; Sigma Aldrich). Control rats were injected with the adjuvant alone. All procedures were performed under isoflurane anesthesia, with buprenorphine $(0.03 \mathrm{mg} / \mathrm{kg})$ being administered for analgesia 2 times/d for $2 \mathrm{~d}$ after immunization. A flow diagram of the study protocol and numbers of rats is shown in Figure 1.

On day 21 after the first immunization, the rats underwent PET imaging with ${ }^{18} \mathrm{~F}-\mathrm{FOL}$. Either contrast-enhanced CT or ${ }^{18} \mathrm{~F}-\mathrm{FDG}$ PET imaging was performed for localization of the myocardium. After PET imaging, the rats were euthanized at $100 \mathrm{~min}$ after injection of ${ }^{18} \mathrm{~F}-\mathrm{FOL}$; blood was drawn by cardiac puncture; and various organs, including the heart, were excised, weighed, and measured for radioactivity using a $\gamma$-counter (Triathler; Hidex) for analysis of tracer biodistribution (15). The left ventricle (LV) was prepared and either embedded in optimal-cuttingtemperature compound and frozen, or fixed in $10 \%$ formaldehyde and embedded in paraffin. The LVs were cut into serial transverse $20-\mu \mathrm{m}$ and $8-\mu \mathrm{m}$ cryosections, or into $4-\mu \mathrm{m}$ paraffin-embedded sections, at 1-mm intervals from base to apex for autoradiography, histology, and immunostainings.

All animal experiments were approved by the national Animal Experiment Board in Finland and the Regional State Administrative Agency for Southern Finland and were conducted in accordance with the relevant European Union directive.

\section{Human Tissue Samples}

Myocardial samples from 5 patients who died of cardiac sarcoidosis, and 1 heart explanted due to sarcoidosis, as described earlier (19), were cut into serial $4-\mu \mathrm{m}$ paraffin sections. Sections were immunohistochemically stained with an antiCD68 antibody to detect macrophages and with an anti-FR- $\beta$ antibody to study localization of FR- $\beta$. Double immunofluorescence staining was performed with an anti-CD68 antibody and with an anti-FR- $\beta$ antibody (20). The use of human 
tissues was in accordance with Finnish law and with the principles of the Declaration of Helsinki and was approved by the National Authority for Medicolegal Affairs and the National Institute for Health and Welfare (19).

\section{Radiotracer}

${ }^{18} \mathrm{~F}-\mathrm{FOL}$ was prepared according to previously described procefrom the end of bombardment. The radiochemical purity was more than $95 \%$, and the molar activity was $34 \pm 9.3 \mathrm{GBq} / \mu \mathrm{mol}$. The radiochemical yields (decay-corrected) were $57 \% \pm 9.2 \%$. In vivo stability of ${ }^{18} \mathrm{~F}-\mathrm{FOL}$ was studied as previously described (15) in veimmunized rats. The fraction of radioactivity related to intact tracer in plasma was $91 \% \pm 2.3 \%$.

\section{In Vivo PET/CT Imaging}

${ }^{18} \mathrm{~F}-\mathrm{FOL}$ in vivo imaging was performed on isoflurane-anesthetized rats using a small-animal PET/CT device (Inveon Multimodality; Siemens Medical Solutions) as previously described $(21,22)$. The rats were injected with $50 \pm 1.5 \mathrm{MBq}$ of ${ }^{18} \mathrm{~F}-\mathrm{FOL}$ via the tail vein. Either a 60-min dynamic PET acquisition (six 10-s, four 60-s, five 300-s, and three 600-s frames) or a 10-min static PET acquisition starting $30 \mathrm{~min}$ after injection was performed.

To visualize the myocardium, a 40 -min static ${ }^{18}$ F-FDG (50 \pm 2.6 $\mathrm{MBq}$ ) PET acquisition starting $20 \mathrm{~min}$ after injection was performed the day before the ${ }^{18} \mathrm{~F}-\mathrm{FOL}$ study. In addition, $300 \mu \mathrm{L}$ of iodinated contrast agent (eXia 160XL; Binitio Biomedical Inc.) was injected intravenously, and high-resolution CT imaging was performed immediately after the ${ }^{18} \mathrm{~F}$-FOL PET as described earlier (21).

Regions of interest of uniform size were defined in the ${ }^{18} \mathrm{~F}-\mathrm{FOL}$ images coregistered with CT or ${ }^{18} \mathrm{~F}$-FDG images using Carimas software (version 2.9; Turku PET Centre) (21). Regions of interest were defined at the $\mathrm{LV}$ myocardium with or without visually increased ${ }^{18} \mathrm{~F}$ FOL uptake, and the $\mathrm{SUV}_{\text {mean }}$ was compared between histologically inflamed and noninflamed myocardial regions as defined by a dures (15). The total radiosynthesis time of was 75-88 min, starting nous blood obtained $80 \mathrm{~min}$ after tracer injection from 3 controls and 4

consensus of 2 readers who were masked to other results. Additional regions of interest were drawn in the liver, lung, kidney, foreleg muscle, inferior vena cava, and LV (blood radioactivity). Decay-corrected time-activity curves were determined in rats using dynamic datasets. To estimate the tracer uptake kinetics, Logan, Patlak, and compartmental modeling was performed, and parametric images were obtained as previously described $(21,22)$.

\section{Ex Vivo Autoradiography, Histology, and Immunostaining}

To confirm uptake of ${ }^{18} \mathrm{~F}-\mathrm{FOL}$ in the myocardium, $20-\mu \mathrm{m}$ cryosections were studied using digital autoradiography as previously described $(21,22)$. The results are expressed as average photo-stimulated luminescence per square millimeter $\left(\mathrm{PSL} / \mathrm{mm}^{2}\right)$ in multiple regions of interest drawn in the histologically inflamed and noninflamed myocardium. For general histology, 20- $\mu \mathrm{m}$ cryosections were stained with hematoxylin and eosin. For immunohistochemistry, adjacent serial $4-\mu \mathrm{m}$ paraffin sections were stained with an anti-FR- $\beta$ antibody, an anti-CD3 antibody to detect lymphocytes, and an anti-CD68 antibody to detect macrophages. Additionally, an anti-iNOS antibody and an anti-CD206 antibody were used to detect proinflammatory (M1-polarized) and antiinflammatory (M2-polarized) macrophages, respectively. To study the colocalization of FR- $\beta$ with macrophage subsets, double immunofluorescence stainings were performed on cryosections. The cryosections were stained with biotinylated anti-FR- $\beta$ (20) antibody detected with streptavidin-Alexa Fluor 488 (Life Technologies Corp.) conjugate followed by staining with either anti-CD68, anti-iNOS, or anti-CD206 antibody (Supplemental Table 1; supplemental materials are available at http://jnm.snmjournals.org).

\section{Effect of Folate Glucosamine on ${ }^{18} \mathrm{~F}-\mathrm{FOL}$ Uptake}

To study the specificity of ${ }^{18} \mathrm{~F}-\mathrm{FOL}$ uptake, an in vivo blocking study was performed with coinjection of a 100-fold excess of the FR- $\beta$ ligand folate glucosamine $\left(\mathrm{C}_{25} \mathrm{H}_{30} \mathrm{~N}_{8} \mathrm{O}_{10} ; 100 \mu \mathrm{L}\right) 10$ min before ${ }^{18} \mathrm{~F}$-FOL injection (Fig. 1). To further evaluate the specificity of the tracer, in vitro binding of ${ }^{18} \mathrm{~F}-\mathrm{FOL}$ in myocardial tissue sections from 3 immunized rats was studied in the presence or absence of a 100-fold excess of folate glucosamine as described earlier (14).

\section{Statistical Analysis}

Data are presented as mean \pm SD. Paired or nonpaired Student $t$ testing was applied to compare normally distributed data. GraphPad Prism, version 6, was used for the statistical analysis, and the threshold for statistical significance was set at a $P$ value of less than 0.05 .

\section{RESULTS}
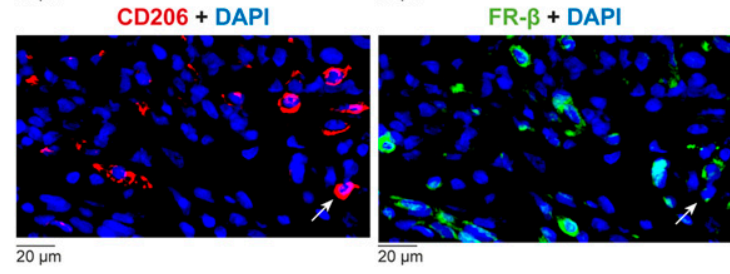

$\overline{20 \mu \mathrm{m}}$

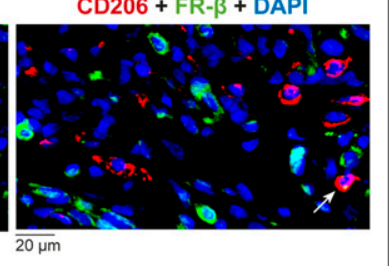

FIGURE 3. Localization of FR- $\beta$ in rat autoimmune myocarditis. Double immunofluorescence staining of sections of inflamed myocardial lesion from immunized rat heart show CD68-positive macrophages, M1-polarized macrophages (iNOS), M2-polarized macrophages (CD206; red, left panels), and FR- $\beta$ (green, middle panels). Note colocalization of FR- $\beta$ with CD68-positive macrophages, and colocalization of M1-polarized macrophages (yellow, right panels) and M2-polarized macrophage (arrows). DAPI = 4',6-diamidino-2-phenylindole.

\section{FR- $\beta$ in Rat Autoimmune Myocarditis}

Histologic analysis of the rat hearts revealed focal cardiac inflammatory lesions in $10(56 \%)$ of 18 immunized rats (6 with hock injections and 4 with footpad injections), whereas no lesions were identified in the 6 control rats (Fig. 2).

The inflammatory lesions were mainly close to the epicardium and showed myocyte necrosis and dense inflammatory cell infiltration. CD68-positive macrophages were the predominant cell type in the lesions and 
consisted of both M1- and M2-polarized macrophages. CD3positive lymphocytes were present to a lesser extent. In immunized rats, the myocardium outside the inflammatory lesions appeared microscopically normal, and CD68 staining was virtually negative. Immunohistochemistry showed abundant FR- $\beta$-positive cells in the lesions but not in the myocardium outside the lesions (Fig. 2).

Double immunofluorescence staining of inflamed myocardial lesions in the rats revealed colocalization of FR- $\beta$ with CD68positive macrophages, which were mainly M1-polarized (Fig. 3).

\section{FR- $\boldsymbol{\beta}$ in Cardiac Sarcoidosis}

Tissue samples of all patients with cardiac sarcoidosis showed FR- $\beta$ expression in the inflammatory lesions that colocalized with CD68-positive macrophages (Fig. 4).

\section{In Vivo PET/CT Imaging of FR- $\beta$}

In vivo imaging demonstrated clearly visible focal ${ }^{18} \mathrm{~F}-\mathrm{FOL}$ uptake in the LV myocardium in all rats with histologically proven inflammatory lesions $(n=7)$ in this subgroup, whereas there was no myocardial tracer uptake in any of the immunized rats without histologic inflammation $(n=8)$ or in the control rats $(n=6$; Fig. 5). Comparisons with histology indicated that ${ }^{18} \mathrm{~F}-\mathrm{FOL}$ uptake was colocalized with myocardial inflammatory lesions, whereas noninflamed myocardium showed very low radioactivity. In total, there were 15 histologically verified separate inflammatory lesions in the 7 rats with myocarditis; all these lesions showed increased ${ }^{18} \mathrm{~F}-\mathrm{FOL}$ uptake.

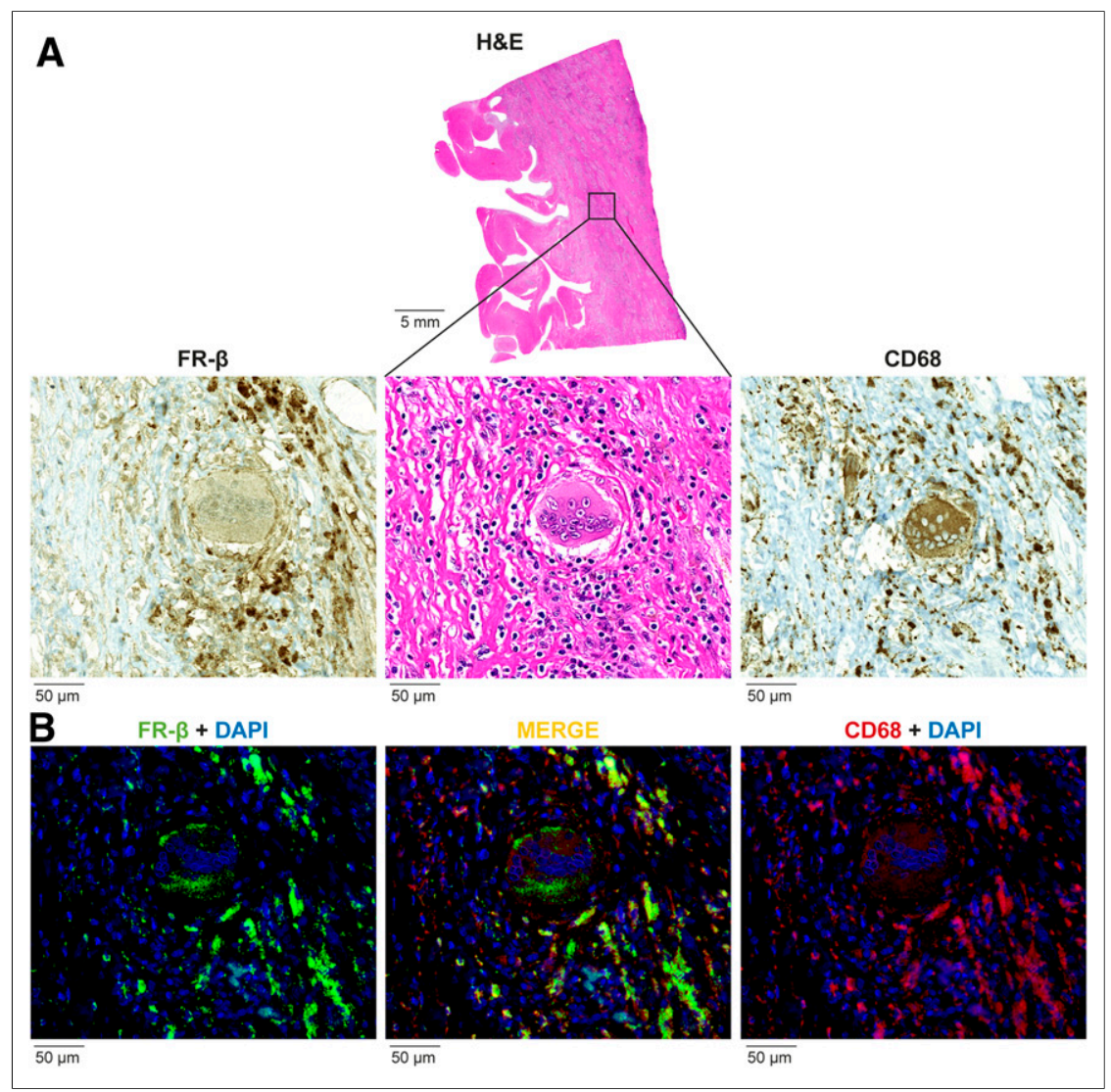

FIGURE 4. FR- $\beta$ in human cardiac sarcoid lesion. (A) Inflammatory lesion identified by hematoxylin and eosin (H\&E) staining and stained with antibodies against CD68-positive macrophages (CD68) and FR- $\beta$. (B) Double immunofluorescence staining with antibodies against CD68-positive macrophages (red, right panel) and FR- $\beta$ (green, left panel), showing colocalization of FR- $\beta$ with CD68-positive macrophages (yellow, middle panel). DAPI = 4',6-diamidino-2-phenylindole.
Time-activity curves in rats with inflamed lesions showed that ${ }^{18} \mathrm{~F}$ FOL radioactivity cleared rapidly from the blood, whereas uptake in myocardial inflammatory lesions and most other tissues remained constant from 20 min after injection (Fig. 6). The highest uptake was observed in the kidneys $\left(\mathrm{SUV}_{\text {mean }}, 10.1 \pm 4.5\right)$, consistent with elimination via the urinary system (Fig. 6).

Figure 6 shows myocardial ${ }^{18} \mathrm{~F}-\mathrm{FOL}$ uptake in PET images at 30-40 min after injection. ${ }^{18} \mathrm{~F}-\mathrm{FOL}$ uptake in the inflammatory lesions was higher than uptake in the noninflamed myocardium of immunized rats $\left(\mathrm{SUV}_{\text {mean }}, 2.1 \pm 1.1\right.$ vs. $\left.0.4 \pm 0.2 ; P=0.004\right)$, with the ratio of ${ }^{18} \mathrm{~F}$-FOL uptake between inflammatory lesions and remote myocardium being around 5:1. The myocardial uptake in nonimmunized controls $\left(\mathrm{SUV}_{\text {mean }}, 0.4 \pm 0.1\right)$ was similar to the noninflamed myocardium of immunized rats $(P=$ $0.4)$ and lower than in inflammatory lesions $(P=0.003)$. Tissues adjacent to the heart showed lower uptake than inflammatory lesions, with an $\mathrm{SUV}_{\text {mean }}$ of $0.3 \pm 0.1$ in $\mathrm{LV}$ blood, $0.3 \pm$ 0.1 in lungs, and $1.2 \pm 0.4$ in liver.

Among the various kinetic models evaluated, the Logan model showed the best fit to the data (Supplemental Figs. 1A and 1B; Supplemental Table 2). Logan plots and parametric images of distribution volume (DV) were consistent with ${ }^{18} \mathrm{~F}$-FOL uptake in the inflamed myocardium (Supplemental Figs. 1A and 1C). The DV of ${ }^{18}$ F-FOL was significantly higher in the inflamed than noninflamed myocardium (DV, $4.0 \pm 1.7$ vs. $0.7 \pm 0.2 ; P=0.004)$ or the myocardium of control rats (DV, $0.8 \pm 0.2 ; P=0.003$; Fig. 6). The $\mathrm{SUV}_{\text {mean }}$ of ${ }^{18} \mathrm{~F}-\mathrm{FOL}$ at $30-40 \mathrm{~min}$ after injection correlated well with DV (Supplemental Fig. 2).

\section{Ex Vivo Autoradiography and Biodistribution}

Autoradiography showed high uptake of ${ }^{18} \mathrm{~F}-\mathrm{FOL}$ in the inflammatory lesions of immunized rats but low uptake in the noninflamed myocardium of immunized rats and the myocardium of control rats (Fig. 7A). The average uptake of ${ }^{18} \mathrm{~F}$ FOL in inflammatory lesions (470 \pm $180 \mathrm{PSL} / \mathrm{mm}^{2} ; n=7$ ) was $7.8 \pm 1.4$-fold higher than that in the noninflamed myocardium of immunized rats $(67 \pm 43$ $\left.\mathrm{PSL} / \mathrm{mm}^{2} ; P=0.0005 ; n=7\right)$. The myocardium of control rats showed low activity $\left(39 \pm 15 \mathrm{PSL} / \mathrm{mm}^{2} ; P=0.0002\right.$ vs. inflamed lesions; $P=0.19$ vs. noninflamed myocardium of immunized rats; $n=6$; Fig. 7B). The biodistribution of ${ }^{18} \mathrm{~F}$-FOL is presented in Supplemental Table 3.

\section{Effect of Folate Glucosamine on ${ }^{18}$ F-FOL Uptake}

In vivo blocking of ${ }^{18} \mathrm{~F}$-FOL with a 100 fold excess of folate glucosamine in $3 \mathrm{im}$ munized rats resulted in $77 \%$ lower ${ }^{18} \mathrm{~F}-\mathrm{FOL}$ uptake in inflammatory lesions than in rats injected with ${ }^{18} \mathrm{~F}-\mathrm{FOL}$ only $\left(\mathrm{SUV}_{\text {mean }}, 0.6 \pm\right.$ 0.1 vs. $2.9 \pm 0.4 ; P=0.02$; Fig. 7 A). Folate glucosamine also effectively reduced in vitro binding of ${ }^{18} \mathrm{~F}-\mathrm{FOL}$ in inflamed myocardium 


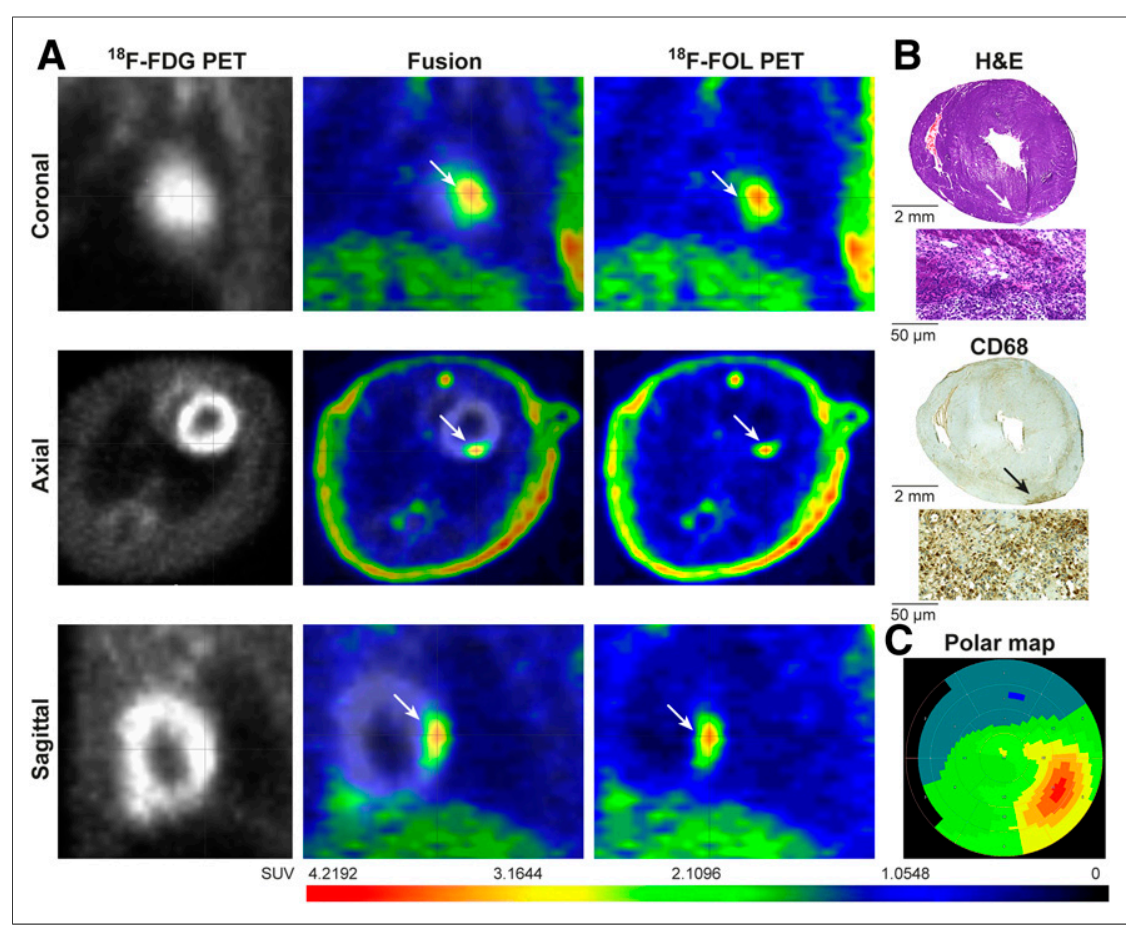

FIGURE 5. (A) In vivo PET images with ${ }^{18} \mathrm{~F}-\mathrm{FDG}$ and ${ }^{18} \mathrm{~F}-\mathrm{FOL}$ tracers in rat with autoimmune myocarditis. PET images at $30-40$ min show focal ${ }^{18} \mathrm{~F}-\mathrm{FOL}$ uptake (arrows) in posterior LV wall. (B) Uptake is colocalized with inflamed myocardial lesion in posterior wall of LV, as shown in histologic sections stained with hematoxylin and eosin (H\&E) or antibodies against CD68 (macrophages). (C) Polar map demonstrates ${ }^{18} \mathrm{~F}-\mathrm{FOL}$ uptake in posterior wall. ${ }^{18} \mathrm{~F}-\mathrm{FOL}$ uptake is low elsewhere in LV myocardium.
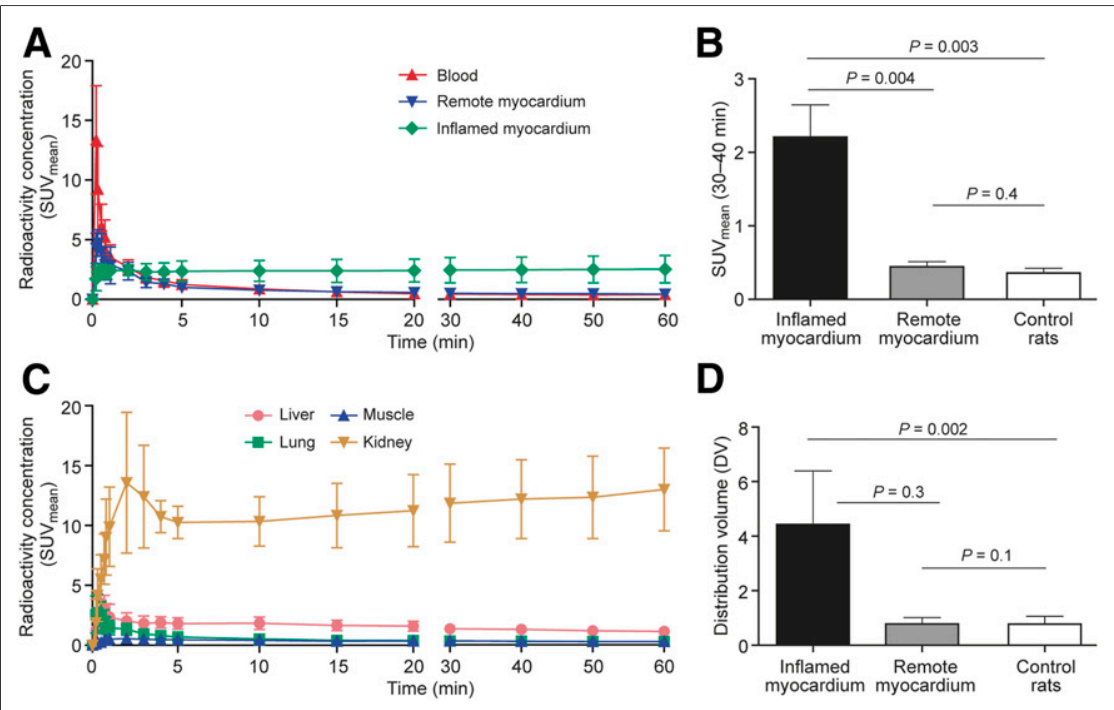

D

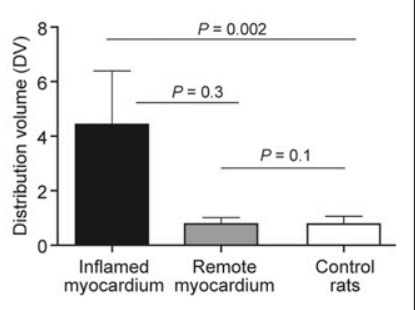

FIGURE 6. PET imaging of ${ }^{18} \mathrm{~F}-\mathrm{FOL}$ uptake. (A and C) Time-activity curves from rats with autoimmune myocarditis $(n=4)$ show that ${ }^{18} \mathrm{~F}-\mathrm{FOL}$ uptake $\left(\mathrm{SUV}_{\text {mean }}\right)$ remains higher in inflamed myocardium than in remote noninflamed myocardium or blood (inferior vena cava). Bars indicate $\mathrm{SD}$. (B) Average myocardial ${ }^{18} \mathrm{~F}-\mathrm{FOL}$ uptake $\left(\mathrm{SUV}_{\text {mean }}\right) 30-40 \mathrm{~min}$ after injection is higher in inflamed myocardium $(n=7)$ than in remote myocardium of immunized rats $(n=7)$ or in myocardium of control rats $(n=6)$. (D) DV indicating that irreversible uptake of ${ }^{18} \mathrm{~F}-\mathrm{FOL}$ is also increased in inflamed myocardium $(n=4)$ in comparison with remote myocardium of immunized rats $(n=4)$ or myocardium of control rats $(n=6)$. Values are mean $\pm \mathrm{SD}$; unpaired $t$ tests were used for comparisons of inflamed and control myocardium, and paired $t$ tests were used for comparisons of inflamed and remote myocardium. in tissue sections $(143 \pm 27$ vs. $2.4 \pm 1.5$ $\mathrm{PSL} / \mathrm{mm}^{2} ; P<0.0001 ; n=3$, Supplemental Fig. 3).

\section{DISCUSSION}

We demonstrate that ${ }^{18} \mathrm{~F}-\mathrm{FOL}$ shows specific uptake in inflamed myocardium in rats with autoimmune myocarditis. ${ }^{18}$ F-FOL PET, as well as ex vivo autoradiography, showed high target-to-background ratios between inflamed lesions and noninflamed myocardium. In the rat model, inflammatory lesions showed high densities of macrophages, particularly M1polarized macrophages expressing FR- $\beta$. Furthermore, macrophages in pathologic specimens of human cardiac sarcoid lesions were shown to express FR- $\beta$. Imaging of FR- $\beta$ expression in inflamed myocardium by ${ }^{18} \mathrm{~F}-\mathrm{FOL}$ PET is a novel approach for the noninvasive assessment of myocarditis.

The rat model of autoimmune myocarditis is a well-established experimental model of myocarditis with similarities to the human giant cell myocarditis leading to inflammatory cardiomyopathy $(17,23,24)$. Although the exact mechanism underlying myocyte injury in autoimmune myocarditis is not fully understood, the disease process is thought to be initiated by autoreactive $\mathrm{T}$ cells and macrophages (17). We studied rats at $21 \mathrm{~d}$ after their first immunization because the peak in inflammation in this model occurs at that time, as shown previously by histology and ${ }^{18}$ F-FDG PET imaging (24,25). Focal myocardial inflammatory lesions characterized by myocyte destruction and by a high prevalence of macrophages (both M1- and M2-polarized) and CD3-positive lymphocytes are typical histologic findings in this model $(23,24)$. We expanded on previous studies by providing evidence that FR- $\beta$ is expressed and colocalized with M1-polarized macrophages in the rat model of myocarditis. Furthermore, we demonstrated that macrophages in human cardiac sarcoid lesions express FR- $\beta$.

Consistent with previous studies (15), our study indicated that ${ }^{18} \mathrm{~F}-\mathrm{FOL}$ shows specific uptake in FR- $\beta$-positive macrophages and favorable kinetics for in vivo imaging of inflammation. We demonstrated that ${ }^{18} \mathrm{~F}$-FOL PET is a potential approach for imaging myocarditis, providing a high target-to-background ratio. Other approaches, including ${ }^{11} \mathrm{C}$-methioninePET (7), 18-kDa translocator proteintargeting PET (11), ${ }^{19} \mathrm{~F}$-based cardiac MR 


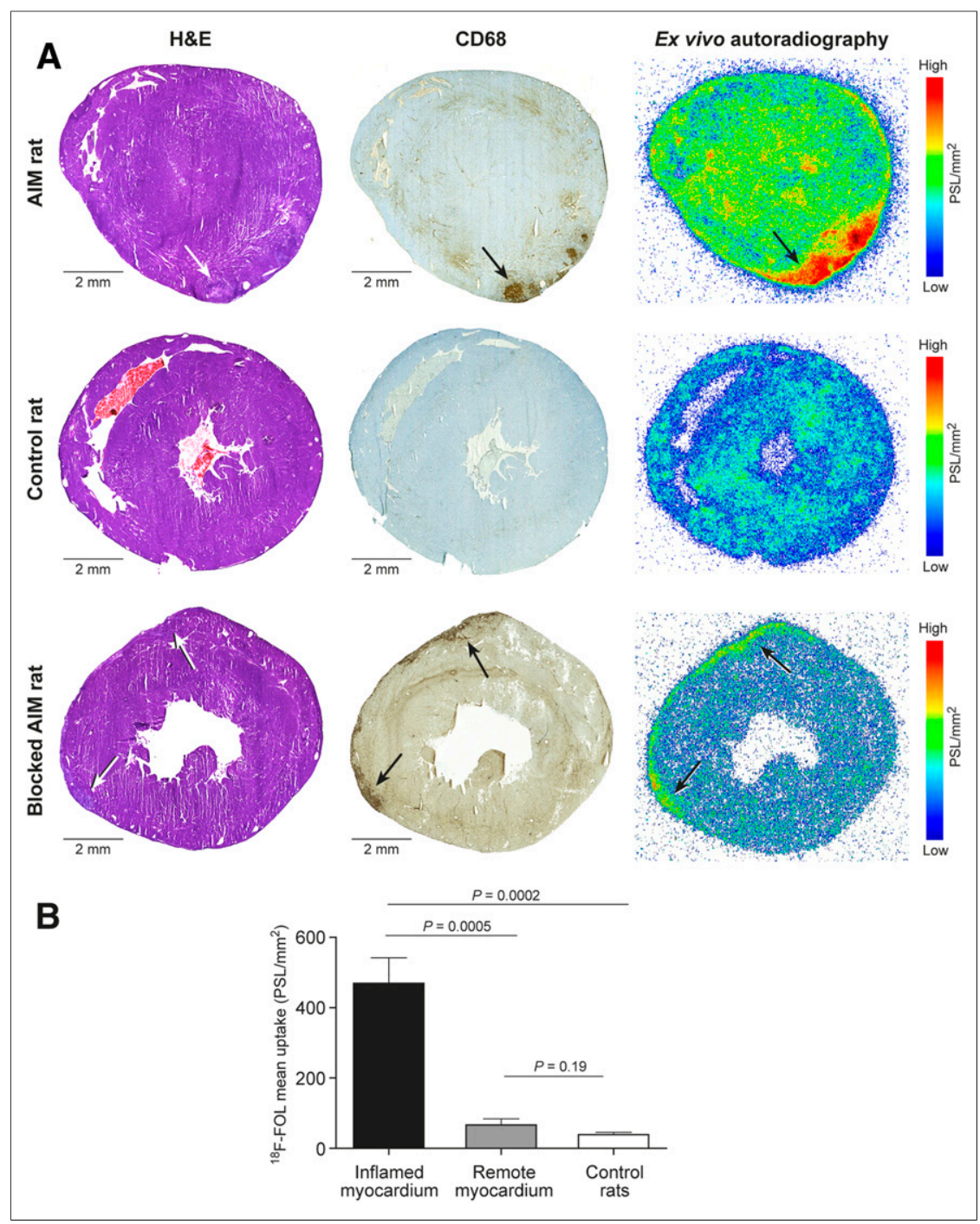

FIGURE 7. Ex vivo autoradiography of ${ }^{18} \mathrm{~F}-\mathrm{FOL}$ uptake. Representative LV short-axis sections stained with hematoxylin and eosin (H\&E) or antibodies against CD68 on macrophages in rat with autoimmune myocarditis (AIM), control rat, and AIM rat after coinjection with 100-fold excess of unlabeled FR- $\beta$ ligand folate glucosamine. Ex vivo autoradiography shows ${ }^{18} \mathrm{~F}-\mathrm{FOL}$ uptake colocalized with inflammatory lesions (black arrows); uptake is reduced by blocking agent. (B) Bars demonstrate ${ }^{18} \mathrm{~F}-\mathrm{FOL}$ uptake by ex vivo autoradiography as mean $\mathrm{PSL} / \mathrm{mm}^{2}$ in inflamed myocardium ( $n=7)$, remote myocardium of immunized rats $(n=7)$, and myocardium of control rats $(n=6)$. Values are mean $\pm \mathrm{SD}$; unpaired $t$ tests were used for comparisons of inflamed and control myocardium, and paired $t$ tests were used for comparisons of inflamed and remote myocardium.

(26), and ${ }^{68} \mathrm{Ga}$-NOTA-mannosylated human serum albumin PET (27), have also shown promising results for the detection of autoimmune myocarditis. Compared with anatomic imaging modalities, molecular imaging has the advantage of more specifically targeting sites of active inflammation. Although direct comparisons between different tracers are not possible, ${ }^{18} \mathrm{~F}$-FOL showed a contrast between inflamed and noninflamed myocardium at least comparable to that previously described for ${ }^{18} \mathrm{~F}-\mathrm{FDG}(3.4 \pm 0.7)$ or ${ }^{11} \mathrm{C}$-methionine $(2.1 \pm 0.2)$ in the same model (7).

Kinetic modeling using graphical Patlak analysis supports the concept of irreversible ${ }^{18} \mathrm{~F}$-FOL uptake in inflamed myocardium, as is consistent with a previous study showing internalization of folate conjugates into endosomes (28). However, the Logan plot showed a better fit to the data, indicating that ${ }^{18} \mathrm{~F}$-FOL uptake may be partly reversible. The observed reversible component might be due to incomplete internalization of the ${ }^{18} \mathrm{~F}-\mathrm{FOL}$, but this remains to be studied.

There are some limitations to our study. We were unable to directly compare uptake of ${ }^{18}$ F-FDG and ${ }^{18}$ F-FOL in inflamed myocardium because of physiologic myocardial ${ }^{18}$ F-FDG uptake. Although our results indicate that $\mathrm{FR}-\beta$ is a potential marker of active myocardial inflammation in both the rat model and human cardiac sarcoidosis, its role in the pathogenesis of myocarditis remains to be studied. Assessing ${ }^{18} \mathrm{~F}-\mathrm{FOL}$ uptake in response to therapy remains to be evaluated in models with a reproducible disease intensity. Notably, FR- $\beta$ can mediate internalization of folate-linked therapeutic agents into cells (29). A FRtargeted imaging agent has been evaluated for tumor imaging in a human study (30). However, evaluation of ${ }^{18} \mathrm{~F}-\mathrm{FOL}$ uptake in human sarcoid lesions was not possible, and therefore, a study with a tracer approved for clinical use will be needed to test the feasibility of imaging FR- $\beta$ in patients with sarcoidosis.

\section{CONCLUSION}

In a rat model of autoimmune myocarditis, ${ }^{18}$ F-FOL PET specifically detects inflamed myocardium containing macrophages expressing FR- $\beta$, which are also present in human cardiac sarcoid lesions. Imaging of FR- $\beta$ expression is a potential approach for the detection of active myocardial inflammation.

\section{DISCLOSURE}

This study was conducted within the Finnish Centre of Excellence in Cardiovascular and Metabolic Diseases supported by the Academy of Finland, University of Turku, Turku University Hospital, and Åbo Akademi University. This study was financially supported by grants from the Academy of Finland, Business Finland, the Jane and Aatos Erkko Foundation, the Finnish Foundation for Cardiovascular Research, and the Sigrid Jusélius Foundation. No other potential conflict of interest relevant to this article was reported.

\section{ACKNOWLEDGMENTS}

We thank Aake Honkaniemi for assistance with the PET studies, Marja-Riitta Kajaala and Erica Nyman (Histology Core, Institute of Biomedicine, University of Turku) for tissue sectioning and immunohistochemical staining, and Timo Kattelus for assistance with figure preparation. 


\section{KEY POINTS}

QUESTION: Can FR- $\beta$-targeted imaging detect myocarditis?

PERTINENT FINDINGS: In this study evaluating whether ${ }^{18} \mathrm{~F}-$ FOL, a PET tracer targeting FR- $\beta$, detects rat autoimmune myocarditis, cardiac inflammatory lesions were detected histologically in 10 of $18(56 \%)$ immunized rats. PET showed specific ${ }^{18} \mathrm{~F}-\mathrm{FOL}$ uptake in inflamed myocardium containing FR- $\beta$-expressing macrophages $\left(S_{U} V_{\text {mean }}, 2.1 \pm 1.1\right.$ vs. $0.4 \pm 0.2$ in noninflamed myocardium; $P<0.01$ ). FR- $\beta$ expressing macrophages were found in human cardiac sarcoidosis specimens.

IMPLICATIONS FOR PATIENT CARE: Current imaging techniques have limited specificity for detecting myocardial inflammation. Imaging of FR- $\beta$-expression is a potential approach for specific detection of active myocardial inflammation.

\section{REFERENCES}

1. Caforio AL, Pankuweit S, Arbustini E, et al. Current state of knowledge on etiology, diagnosis, management, and therapy of myocarditis: a position statement of the European Society of Cardiology Working Group on Myocardial and Pericardial Diseases. Eur Heart J. 2013;34:2636-2648.

2. Kadkhodayan A, Chareonthaitawee P, Raman SV, Cooper LT. Imaging of inflammation in unexplained cardiomyopathy. JACC Cardiovasc Imaging. 2016; 9:603-617.

3. Friedrich MG, Sechtem U, Schulz-Menger J, et al. Cardiovascular magnetic resonance in myocarditis: a JACC white paper. J Am Coll Cardiol. 2009;53:1475-1487.

4. Youssef G, Leung E, Mylonas I, et al. The use of ${ }^{18} \mathrm{~F}-\mathrm{FDG}$ PET in the diagnosis of cardiac sarcoidosis: a systematic review and metaanalysis including the Ontario experience. J Nucl Med. 2012;53:241-248.

5. Blankstein R, Osborne M, Naya M, et al. Cardiac positron emission tomography enhances prognostic assessments of patients with suspected cardiac sarcoidosis. J Am Coll Cardiol. 2014;63:329-336.

6. Tang R, Wang JT, Wang L, et al. Impact of patient preparation on the diagnostic performance of ${ }^{18}$ F-FDG PET in cardiac sarcoidosis: a systematic review and meta-analysis. Clin Nucl Med. 2016;41:e327-e339.

7. Maya Y, Werner RA, Schütz C, et al. ${ }^{11} \mathrm{C}$-methionine PET of myocardial inflammation in a rat model of experimental autoimmune myocarditis. $\mathrm{J}$ Nucl Med. 2016;57:1985-1990.

8. Martineau P, Pelletier-Galarneau M, Juneau D, et al. Imaging cardiac sarcoidosis with FLT-PET compared with FDG/perfusion-PET: a prospective pilot study. JACC Cardiovasc Imaging. 2019;12:2280-2281.

9. Lapa C, Reiter T, Kircher M, et al. Somatostatin receptor based PET/CT in patients with the suspicion of cardiac sarcoidosis: an initial comparison to cardiac MRI. Oncotarget. 2016;7:77807-77814.

10. Gormsen LC, Haraldsen A, Kramer S, Dias AH, Kim WY, Borghammer P. A dual tracer ${ }^{68} \mathrm{Ga}$-DOTANOC PET/CT and ${ }^{18} \mathrm{~F}$-FDG PET/CT pilot study for detection of cardiac sarcoidosis. EJNMMI Res. 2016;6:52.

11. Kim GR, Paeng JC, Jung JH, et al. Assessment of TSPO in a rat experimental autoimmune myocarditis model: a comparison study between $\left[{ }^{18} \mathrm{~F}\right]$ fluoromethylPBR28 and $\left[{ }^{18} \mathrm{~F}\right] \mathrm{CB} 251$. Int $J$ Mol Sci. 2018;19:276.
12. Lapa C, Reiter T, Li X, et al. Imaging of myocardial inflammation with somatostatin receptor based PET/CT: a comparison to cardiac MRI. Int J Cardiol. 2015;194:44-49.

13. Paulos CM, Turk MJ, Breur GJ, Low PS. Folate receptor-mediated targeting of therapeutic and imaging agents to activated macrophages in rheumatoid arthritis. Adv Drug Deliv Rev. 2004;56:1205-1217.

14. Hu Y, Wang B, Shen J, et al. Depletion of activated macrophages with a folate receptor-beta-specific antibody improves symptoms in mouse models of rheumatoid arthritis. Arthritis Res Ther. 2019;21:143.

15. Silvola JMU, Li X, Virta J, et al. Aluminum fluoride-18 labeled folate enables in vivo detection of atherosclerotic plaque inflammation by positron emission tomography. Sci Rep. 2018;8:9720.

16. Ni NC, Jin CS, Cui L, et al. Non-invasive macrophage tracking using novel porphysome nanoparticles in the post-myocardial infarction murine heart. $\mathrm{Mol}$ Imaging Biol. 2016;18:557-568.

17. Kodama M, Matsumoto Y, Fujiwara M, Masani F, Izumi T, Shibata A. A novel experimental model of giant cell myocarditis induced in rats by immunization with cardiac myosin fraction. Clin Immunol Immunopathol. 1990;57:250-262.

18. Kamala T. Hock immunization: a humane alternative to mouse footpad injections. J Immunol Methods. 2007;328:204-214.

19. Ekström K, Lehtonen J, Nordenswan HK, et al. Sudden death in cardiac sarcoidosis: an analysis of nationwide clinical and cause-of-death registries. Eur Heart J. 2019;40:3121-3128.

20. Shen J, Putt KS, Visscher DW, et al. Assessment of folate receptor- $\beta$ expression in human neoplastic tissues. Oncotarget. 2015;6:14700-14709.

21. Ståhle M, Kytö V, Kiugel M, et al. Glucagon-like peptide-1 receptor expression after myocardial infarction: imaging study using ${ }^{68} \mathrm{Ga}-\mathrm{NODAGA}-\mathrm{exendin}-4$ positron emission tomography. J Nucl Cardiol. December 13, 2018 [Epub ahead of print].

22. Kiugel M, Dijkgraaf I, Kytö V, et al. Dimeric $\left[{ }^{68} \mathrm{Ga}\right.$ ]DOTA-RGD peptide targeting $\alpha v \beta 3$ integrin reveals extracellular matrix alterations after myocardial infarction. Mol Imaging Biol. 2014;16:793-801.

23. Kodama M, Zhang S, Hanawa H, Shibata A. Immunohistochemical characterization of infiltrating mononuclear cells in the rat heart with experimental autoimmune giant cell myocarditis. Clin Exp Immunol. 1992;90:330-335.

24. Okura Y, Yamamoto T, Goto S, et al. Characterization of cytokine and iNOS mRNA expression in situ during the course of experimental autoimmune myocarditis in rats. J Mol Cell Cardiol. 1997;29:491-502.

25. Werner RA, Wakabayashi H, Bauer J, et al. Longitudinal ${ }^{18} \mathrm{~F}-\mathrm{FDG}$ PET imaging in a rat model of autoimmune myocarditis. Eur Heart $J$ Cardiovasc Imaging. 2019;20:467-474.

26. van Heeswijk RB, De Blois J, Kania G, et al. Selective in vivo visualization of immune-cell infiltration in a mouse model of autoimmune myocarditis by fluorine-19 cardiac magnetic resonance. Circ Cardiovasc Imaging. 2013;6:277-284.

27. Lee SP, Im HJ, Kang S, et al. Noninvasive imaging of myocardial inflammation in myocarditis using ${ }^{68} \mathrm{Ga}$-tagged mannosylated human serum albumin positron emission tomography. Theranostics. 2017;7:413-424.

28. Bandara NA, Hansen MJ, Low PS. Effect of receptor occupancy on folate receptor internalization. Mol Pharm. 2014;11:1007-1013.

29. Xia W, Hilgenbrink AR, Matteson EL, Lockwood MB, Cheng JX, Low PS. A functional folate receptor is induced during macrophage activation and can be used to target drugs to activated macrophages. Blood. 2009;113:438-446.

30. Fisher RE, Siegel BA, Edell SL, et al. Exploratory study of ${ }^{99 \mathrm{~m}} \mathrm{Tc}-\mathrm{EC} 20$ imaging for identifying patients with folate receptor-positive solid tumors. J Nucl Med. 2008;49:899-906. 